\title{
Diagnóstico e Quantificação de Doenças Fúngicas da Acerola no Estado da Paraíba
}

\author{
Fernandes A. Almeida ${ }^{1}$, Egberto Araújo ${ }^{2}$, Hermes Gonçalves Junior ${ }^{1}$, Artur F. Barreto ${ }^{1} \&$ Renato \\ A. G. Carvalho ${ }^{1}$ \\ ${ }^{1}$ Programa de Pós-Graduação em Agronomia; ${ }^{2}$ Departamento de Fitotecnia, Centro de Ciências Agrárias, Universidade \\ Federal da Paraíba, Cx. Postal 42, CEP 58397-970, Areia, PB, e-mail: egberto@ cca.ufpb.br
}

(Aceito para publicação em 20/11/2002)

Autor para correspondência: Egberto Araújo

ALMEIDA, F.A., ARAÚJO, E., GONÇALVES JUNIOR, H., BARRETO, A.F. \& CARVALHO, R.A.G. Diagnóstico e quantificação de doenças da acerola no Estado da Paraíba. Fitopatologia Brasileira 28:176-179. 2003.

\section{RESUMO}

Objetivando o diagnóstico e quantificação das doenças da acerola (Malpighia emarginata) no Estado da Paraíba, realizou-se um levantamento nas microrregiões do Sertão, Curimatau, Brejo e Litoral. As doenças identificadas foram a cercosporiose (Cercospora sp.), fusariose (Fusarium oxysporum), fumagina (Capnodium sp.), antracnose (Colletotrichum gloeosporioides) e mancha de Alternaria (Alternaria sp.). Além dessas, no teste de patogenicidade, verificou-se o desenvolvimento de lesões circulares, secamento e queda de folhas que foram previamente feridas e inoculadas com um fungo do gênero Pestalotiopsis, isolado de material doente coletado em áreas de plantio. Nas microrregiões do Brejo e Curimatau verificaram-se, respectivamente, o maior e o menor número de doenças. A cercosporiose ocorreu em todas as microrregiões, enquanto a antracnose e a fumagina foram verificadas no Brejo e Litoral, e a fusariose nas áreas irrigadas do Sertão e Curimataú. No Sertão e no Brejo, ocorreu, ainda, a mancha de Alternaria e pestalotiose, respectivamente. Os maiores valores de incidência e severidade da fumagina, antracnose e cercosporiose foram associados a elevações da precipitação pluviométrica nas microrregiões em que foram assinaladas, como também a presença de insetos sugadores para a primeira doença.

Palavras-chave adicionais: Malpighia emarginata, incidência e severidade de doenças.

\section{ABSTRACT}

Diagnostic and quantification of barbados cherry diseases in the State of Paraíba, Brazil

This work was aimed at diagnosing and quantificating barbados cherry (Malpighia emarginata) fungal diseases in different micro-regions of Paraíba. Black mould, anthracnose and alternaria leaf spot were observed in the plant material sampled caused, respectivelly, by Cercospora sp., Fusarium oxysporum, Capnodium sp., Colletotrichum gloeosporioides and Alternaria sp. The pathogenicity test performed showed the development of round lesions and the drying and falling of punctured leaves inoculated with a fungus of genus Pestalotiopsis isolated from material naturally infected. The greates and least number of diseases were observed in the microregions of Brejo and Curimatau, respectively. Cercospora leaf spot occurred in all localities. Anthracnose and black mould were observed in Brejo and Litoral micro-regions, and the fusarium wilt occurried in Sertão and Curimatau micro-regions. Alternaria occurred in the Sertão and pestalotia leaf spot occurred in Brejo. The higher values of incidence and severity for anthracnose, black mould and pestalotia leaf spot were associated with increased rainfall. For anthracnose, the presence of sucker insects was also higher.
A aceroleira (Malpighia emarginata DC.) vem sendo cultivada amplamente no Estado da Paraíba, tanto em áreas litorâneas quanto no interior. Considerando-se que essa é uma cultura introduzida e que se expandiu rapidamente, justificase a necessidade do conhecimento das doenças que estão ocorrendo e a intensidade com que se verificam.

Em relação a essa cultura são relatadas diversas doenças fúngicas, destacando-se a antracnose causada por Colletotrichum gloeosporioides (Penz.) Penz. \& Sacc. e $C$. dematium (Pers. ex Fr.) Grove que, segundo Alves et al.
(1995), é a doença mais difundida no Brasil. No Nordeste, em pomares de acerola situados em áreas irrigadas do vale do São Francisco, foram constatados os fungos Fusarium oxysporum Schlecht, causando seca rápida de toda planta e levando à morte, e Botryodiplodia theobromae Pat., que reduz o vigor, e provoca secamento descendente (Tavares, 1995). Na Ilha de São Luís, Estado do Maranhão, Silva et al. (1997) isolaram da folhagem de acerola e comprovaram a patogenicidade de Corynespora cassiicola (Berk. \& Curt.) Wei. No estado do Ceará, Holanda et al. (1997) realizaram o 
Diagnóstico e quantificação de doenças fúngicas da acerola no Estado...

mapeamento das doenças da aceroleira nas zonas fisiográficas do Sertão, Litoral e Serras Úmidas, sendo constatado a podridão dos frutos e a podridão seca das hastes causadas, respectivamente, por Rhizopus nigricans Ehr. e Lasiodiplodia theobromae (Pat) Griff. \& Maubl. (=Botryodiplodia theobromae Pat.). Em levantamento efetuado nos cerrados do Distrito Federal, Goiás, Minas Gerais e Mato Grosso foram constatados danos consideráveis causados pelos fungos Cercosporidium sp. e C. gloeosporioides, provocando queda de frutos e flores e lesões necróticas profundas; Cladosporium herbarum (Pers.) Link ex. Fr., danificando frutos em fase de pré-colheita; B. theobromae e Phomopsis sp., causando podridão da casca do tronco e Cylindrocladium clavatum Hodges \& May, causando podridão das raízes e lesões no coleto (Urben et al.,1996).

$\mathrm{Na}$ primeira fase desse estudo, entre junho e novembro de 1996, realizou-se o diagnóstico a partir de material com sintomas de doenças, coletado nas microrregiões do Sertão do Baixo Piranhas, Curimatau, Brejo e Litoral do estado da Paraíba. Além da visualização do quadro sintomatológico e dos sinais, realizaram-se isolamentos de fungos e testes de patogenicidade, conforme os procedimentos usuais em estudos dessa natureza (French \& Hebert, 1982; Dhingra e Sinclair, 1985).

Após o diagnóstico foram realizadas visitas trimestrais, entre janeiro de 1997 e junho de 1998, aos campos de produção, para a avaliação das doenças. Os dados climáticos, referentes à precipitação pluviométrica acumulada trimestralmente, respectivamente para as microrregiões do Sertão, Curimatau, Brejo e Sertão, foram os seguintes: primeiro trimestre $-147 \mathrm{~mm}, 12 \mathrm{~mm}, 0 \mathrm{~mm}$ e $89 \mathrm{~mm}$; segundo trimestre $-37 \mathrm{~mm}, 11 \mathrm{~mm}, 244 \mathrm{~mm}$ e $27 \mathrm{~mm}$; terceiro trimestre $-0 \mathrm{~mm}, 11 \mathrm{~mm}, 244 \mathrm{~mm}$ e $27 \mathrm{~mm}$; e, quarto trimmestre $-0 \mathrm{~mm}, 37 \mathrm{~mm}, 0 \mathrm{~mm}$ e $91 \mathrm{~mm}$. Nas inspeções de campo foram determinadas a incidência (percentagem de plantas atacadas) da fusariose $(F$. oxysporum), e as incidência (percentagem de folhas atacadas) e severidade (extensão da doença) da antracnose (C. gloeosporioides), cercosporiose (Cercospora sp), fumagina (Capnodium sp.), mancha de alternaria (Alternaria sp.) e pestalotiose (Pestalotiopsis). Nas avaliações da extensão da antracnose, cercosporiose, mancha de alternaria e pestalotiose foi empregada a seguinte escala: nota 0 - folha sadia; nota 1 - de uma a três lesões puntiformes por folha; nota 2 - de quatro a cinco lesões puntiformes; nota 3 - de quatro a cinco pequenas lesões circulares; nota 4 lesões maiores que $5 \mathrm{~mm}$ de diâmetro, isoladas e confluentes; nota 5 - lesões confluentes ocupando mais da metade das folhas. Para a fumagina, avaliou-se a extensão do crescimento fúngico, escuro, sobre os limbos foliares, atribuindo-se as seguintes notas: $0=$ ausência de fungo; 1 = uma a três colônias pequenas (= $5 \mathrm{~mm}$ de diâmetro); $2=$ mais de três colônias pequenas, isoladas, cobrindo parcialmente o limbo foliar; $3=$ colônias maiores que 5 mm de diâmetro, confluentes, cobrindo parcialmente o limbo foliar; e, 4 = colônias confluentes constituindo uma lâmina, cobrindo totalmente o limbo. A severidade de todas as doenças foi calculada empregando-se o Índice de McKinney (Chester, 1950): $\mathrm{S}=\sum \mathrm{f}(\mathrm{N}) / \mathrm{NTF} \times \mathrm{NM}$, sendo $\mathrm{S}$ = Severidade; $\sum \mathrm{F}(\mathrm{N})=$ somatório da freqüência de notas; $\mathrm{NTF}=$ número total de folhas examinadas; $\mathrm{e}, \mathrm{NM}=$ nota máxima da escala.

Das doenças constatadas, a antracnose, cercosporiose, mancha de alternaria e fusariose já foram referenciadas ocorrendo na aceroleira. (Carvalho, 1992; Gonzaga Neto \& Soares, 1994; Freire, 1995; Alves et al., 1995; Tavares, 1995; Urben et al., 1996). No entanto, em lesões foliares e do caule, foi isolado um fungo do gênero Pestalotiopsis (Sutton, 1980) que, no teste de patogenicidade, no oitavo dia após a inoculação do mesmo em folhas previamente feridas com bisturí, foram observados sintomas caracterizados por manchas necróticas circulares, de coloração castanho-escura, medindo de 3 a $5 \mathrm{~mm}$ de diâmetro, distribuídas por todo o limbo, seguindo-se o rápido secamento e queda das folhas; nos ramos não foram observados sintomas. As lesões decorrentes da ação de fungos do gênero Pestalotiopsis são consideradas de importância secundária e a penetração desses organismos, geralmente, dá-se por meio de aberturas naturais, ferimentos ou de lesões provocadas por outros patógenos (Menezes, 1997; Furtado, 1997).

A ocorrência das doenças da aceroleira variou conforme as microrregiões do Estado da Paraíba e os períodos de observações (Figuras 1 e 2). Foi na microregião do Brejo que se observou o maior número de doenças, sendo constatadas a cercosporiose, fumagina, antracnose e pestalotiose; as três primeiras doenças também ocorreram no Litoral. No Sertão, verificou-se a ocorrência da cercosporiose, fusariose e mancha de Alternaria e no Curimataú, a cercosporiose e fusariose nos primeiro, terceiro e quarto trimestres de observações.

A cercoporiose foi constatada em todas as microrregiões, mas não em todos os períodos de observações. A maior severidade dessa doença se verificou no Brejo, durante o segundo trimestre. Não foram verificados sintomas de cercosporiose nas observações realizadas nos primeiro, segundo e terceiro semestres, respectivamente, nas microrregiões do Brejo, Curimataú e Sertão do Baixo Piranhas (Figuras 1 e 2), quando as precipitações registradas nas mesmas foram nulas ou mínimas (0-11 mm).

Nas microrregiões do Brejo e Litoral, onde foram verificadas a antracnose na aceroleira, registraram-se as precipitações mais elevadas e, provavelmente, em relação ao Sertão do Baixo Piranhas e o Curimataú, devem ter apresentado maiores valores de umidade relativa e temperaturas mais baixas, condições que favorecem a ocorrência dessas doenças (Agrios, 1988; Ribeiro, 1997). As condições climáticas prevalecentes no Brejo e Litoral devem também ter favorecido uma maior atividade de insetos sugadores (cochonilhas, pulgões, trips, etc.) que, atacando as plantas proporcionaram o substrato para os agentes causais da fumagina (Agrios, 1988).

Para as doenças que ocorreram no Curimataú e Sertão 
F.A. Almeida et al.

\section{INCIDÊNCIA}
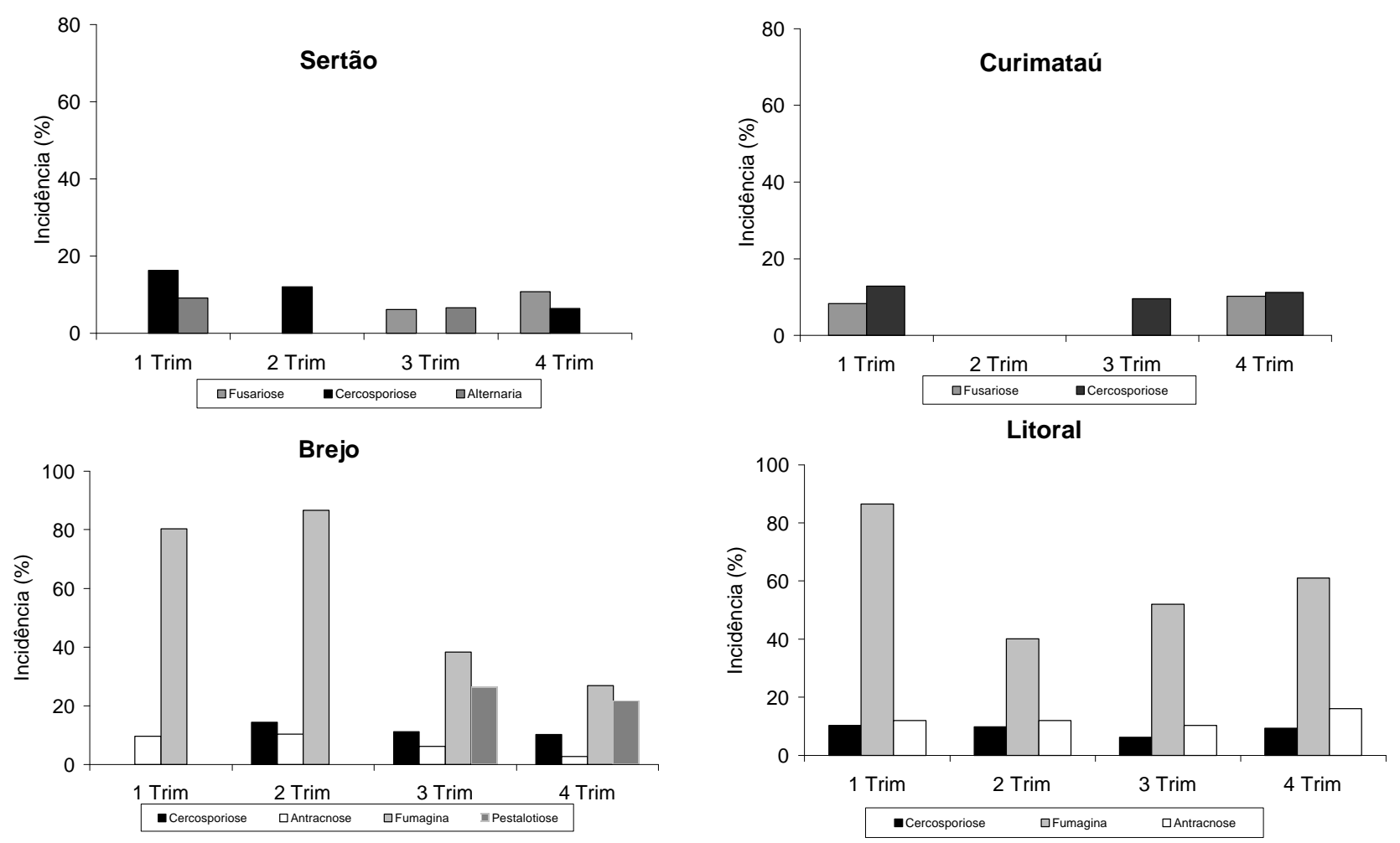

FIG. 1 - Incidência de doenças da aceroleira (Malpighia emarginata) em quatro microrregiões do Estado da Paraíba

\section{SEVERIDADE}
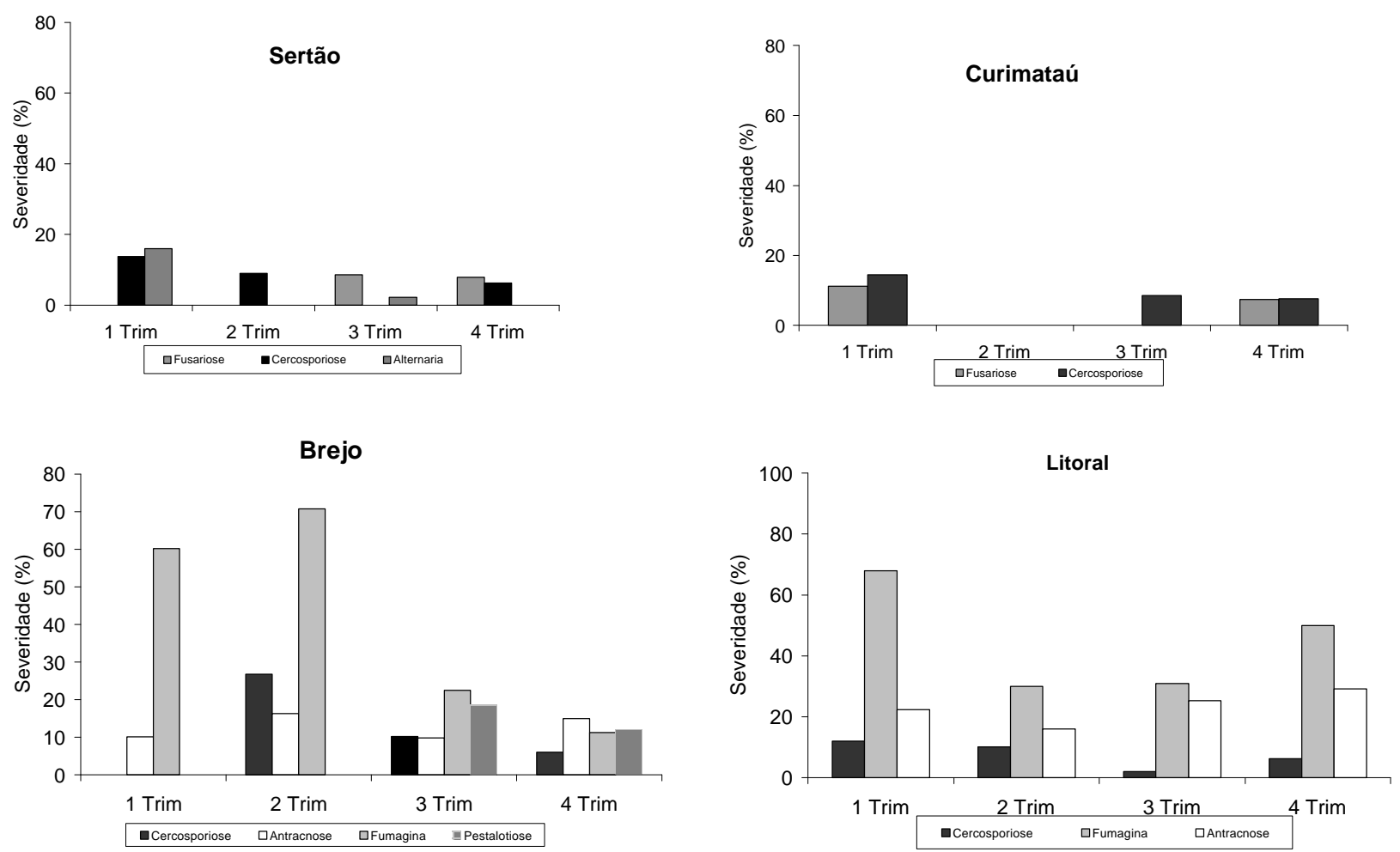

FIG. 2 - Severidade de doenças da aceroleira (Malpighia emarginata) em quatro microrregiões do estado da Paraíba. 
Diagnóstico e quantificação de doenças fúngicas da acerola no Estado...

do Baixo Piranhas, os valores de incidência e severidade foram aproximados; a primeira microregião foi a menos chuvosa e, na segunda microregião não foram registradas precipitações pluviométricas nos terceiro e quarto semestres, sendo as plantações mantidas precariamente por meio de irrigações.

\section{REFERÊNCIAS BIBLIOGRÁFICAS}

AGRIOS, G.N. Plant Pathology. $3^{\text {rd }}$ ed. New York. Academic Press. 1988.

ALVES, R.E., MENEZES, J.B. \& SILVA, S.M. Colheita e pós-colheita de acerola. In: São José, A.R. \& Alves, R.E. (Eds.) Acerola no Brasil: Produção e Mercado. Vitória da Conquista - BA, 1995. pp.77-89.

CHESTER, K.S. Plant disease losses: their appraisal and interpretation. Plant Disease Reporter 193:190-362. 1950.

DHINGRA, O.D. \& SINCLAIR, J.B. Basic plant pathology methods. Boca Raton, Florida. CRC Press. 1985.

FRENCH, E.R. \& HEBERT, T.T. Métodos de Investigación Fitopatológica. San José, Costa Rica. IICA. 1982.

FREIRE, F.C.O. Doenças da Acerola no Brasil. In: São José, A.R. \& Alves, R.E. (Eds.) Acerola no Brasil: Produção e Mercado. Vitória da Conquista - BA. 1995. pp.71-76.

FURTADO, E.L. Doenças do chá. In: Kimati, H., Amorim, L., Bergamim Filho, A., Camargo, L.E.A. \& Rezende, J.A.M. (Eds.)
Manual de Fitopatologia; Doenças de Plantas. 3ㄹ ed. São Paulo. Agronômica Ceres. 1997. pp.257-260.

GONZAGA NETO, L. \& SOARES, J.M. Acerola para exportação: aspectos técnicos da produção. Brasília-DF. EMBRAPA/SPI. Série Publicações Técnicas FRUPEX, 14. 1994.

HOLANDA, Y.C.A., PONTES, J.J. da \& SILVEIRA FILHO, J. Doenças da acerola (Malpighia glabra L.) no Estado do Ceará, Brasil. Fitopatologia Brasileira 22:453. 1997.

MENEZES, M. Doenças do Cajueiro. In: Kimati, H., Amorim, L., Bergamim Filho, A., Camargo, L.E.A. \& Rezende, J.A.M. (Eds.) Manual de Fitopatologia; Doenças de Plantas. 3르 ed. São Paulo. Agronômica Ceres. 1997. pp.201-206.

RIBEIRO, I.J.A. Doenças da mangueira. In: Kimati, H., Amorim, L., Bergamim Filho, A., Camargo, L.E.A. \& Rezende, J.A.M. (Eds.) Manual de Fitopatologia; Doenças de Plantas. 3a ed. São Paulo. Agronômica Ceres. 1997. pp.511-524.

SILVA, G.S. da; RODRIGUES, A.A.C. \& SOARES JUNIOR, A.C. Mancha de Corynespora em acerola (Malpighia glabra L.). Fitopatologia Brasileira 22:452. 1997 (Resumo).

SUTTON, B.C. The Coelomycetes. Kew. CMI. 1980.

TAVARES, S.C.H. Doenças da acerola no Brasil. Petrolina - PE. EMBRAPA/CPATSA. 1995.

URBEN, A.F., JUNQUEIRA, N.T.V., RAMOS, V.H.V. \& OLIVEIRA, M.A.S. Doenças da acerola e de frutos nos cerrados da região centro oeste e Minas Gerais. Fitopatologia Brasileira 21:387. 1996. (Resumo). 